(2)

\title{
Feasibility and effectiveness of a disease and care management model in the primary health care system for patients with heart failure and diabetes (Project Leonardo)
}

This article was published in the following Dove Press journal:

Vascular Health and Risk Management

27 April 2010

Number of times this article has been viewed

\section{Marco Matteo Ciccone \\ Ambrogio Aquilino ${ }^{2}$ \\ Francesca Cortese' \\ Pietro Scicchitano' \\ Marco Sassara' \\ Ernesto Mola ${ }^{3}$ \\ Rodolfo Rollo ${ }^{4}$ \\ Pasquale Caldarola ${ }^{5}$ \\ Francesco Giorgino 6 \\ Vincenzo Pomo ${ }^{2}$ \\ Francesco Bux ${ }^{2}$ \\ 'Section of Cardiovascular Disease, Department of Emergency and Organ Transplantation, School of Medicine, University of Bari, Bari, Italy; ${ }^{2}$ Agenzia Regionale Sanitaria - Regione Puglia (ARES), Apulia, Italy; ${ }^{3} \mathrm{ASL}$, Lecce, Italy; ${ }^{4} \mathrm{ASL}$, Brindisi, Italy; ${ }^{5} \mathrm{Cardiologia}$, Ospedale "Sarcone", Terlizzi, Italy; "Section of Endocrinology, \\ Department of Emergency and Organ Transplantation, School of Medicine, University of Bari, Bari, Italy}

Correspondence: Marco Matteo Ciccone Institute of Cardiology, University of Bari, Policlinico, Piazza Giulio Cesare II,

70124 Bari, Italy

Tel +39080 5478622

Fax +39080 5478796

Email ciccone@cardio.uniba.it
Purpose: Project Leonardo represented a feasibility study to evaluate the impact of a disease and care management (D\&CM) model and of the introduction of "care manager" nurses, trained in this specialized role, into the primary health care system.

Patients and methods: Thirty care managers were placed into the offices of 83 general practitioners and family physicians in the Apulia Region of Italy with the purpose of creating a strong cooperative and collaborative "team" consisting of physicians, care managers, specialists, and patients. The central aim of the health team collaboration was to empower 1,160 patients living with cardiovascular disease (CVD), diabetes, heart failure, and/or at risk of cardiovascular disease (CVD risk) to take a more active role in their health. With the support of dedicated software for data collection and care management decision making, Project Leonardo implemented guidelines and recommendations for each condition aimed to improve patient health outcomes and promote appropriate resource utilization.

Results: Results show that Leonardo was feasible and highly effective in increasing patient health knowledge, self-management skills, and readiness to make changes in health behaviors. Patient skill-building and ongoing monitoring by the health care team of diagnostic tests and services as well as treatment paths helped promote confidence and enhance safety of chronic patient management at home.

Conclusion: Physicians, care managers, and patients showed unanimous agreement regarding the positive impact on patient health and self-management, and attributed the outcomes to the strong "partnership" between the care manager and the patient and the collaboration between the physician and the care manager. Future studies should consider the possibility of incorporating a patient empowerment model which considers the patient as the most important member of the health team and care managers as key health care collaborators able to enhance and support services to patients provided by physicians in the primary health care system.

Keywords: partnerships, health team, patient empowerment, care coordination

\section{Introduction}

According to the World Health Organization, CVD is the leading cause of death worldwide accounting for approximately 18 million deaths a year or $33 \%$ of the 55 million deaths annually. ${ }^{1,2}$ Age, especially for people aged 65 years or older, increases the risks associated with heart disease; the likelihood of developing CVD, suffering a coronary event such as a heart attack or stroke, or death from heart disease is significantly greater. Currently, at a global level, $10.7 \%$ of men and $14.7 \%$ of women are

submit your manuscript $\mid$ www.dovepress.con 
over the age of 65 . Italy has one of the oldest populations in the world, with $14.6 \%$ of men and $19.8 \%$ of women aged 65 years or older. ${ }^{2,3}$ In Europe, CVD represents the main cause of morbidity, mortality, and hospitalization costing countries $€ 190$ billion yearly., ${ }^{4,5}$

Addressing this increased incidence of chronic disease is one of the most important challenges for the health system. In contrast to the traditional "medical model" management of acute conditions, characterized by a short period of patient compliance with following the doctor's orders, management of a chronic disease requires that patients take a more active role in the day-to-day decisions about the management of their illness. ${ }^{6,7}$ This new disease paradigm requires that there be a working patient-provider "partnership" that involves effective treatment within an integrated system of collaborative care which includes self-management education and follow-up. ${ }^{8}$ Both patient and provider roles need to be modified in the treatment of a chronic condition. Patients are expected to do what is needed to manage the condition on a daily basis; health care providers act as consultants, interpreters of symptoms, resource persons, and offer treatment suggestions..$^{9,10}$

The Chronic Care Model developed by Wagner et al, ${ }^{11}$ and the Innovative Care for Chronic Conditions, ${ }^{12}$ edited by the World Health Organization, propose that ideal care for chronic conditions is achieved when health care providers interact with informed patients. The essential ingredient of effective chronic care treatment is the partnership between the patient and health professionals because it offers the opportunity to empower patients to become more active in managing their health. When patients are more informed, involved, and empowered, they interact more effectively with health care providers and strive to take actions that will promote healthier outcomes. ${ }^{8,11}$ In addition, the partnership between the patient and health professional allows for the care plan to be individualized and to address the specific knowledge patients must have and the behaviors they must change to manage the condition effectively. Patients are supported in self-management education that is focused on providing them with the skills to live as active and meaningful a life as possible with their chronic condition. The patient is central to defining the "disease-related problems" and the self-management program assists them with problem solving and gaining the self-efficacy and confidence to deal with these problems. ${ }^{10,13}$ Evidence suggests that when programs teaching self-management consider the patient's assessment of their condition, there is greater patient satisfaction with care, better patient compliance with treatment, and higher likelihood of maintenance of continuous relationships in health care. ${ }^{14}$

Project Leonardo provided proactive identification and treatment of patients with diabetes, heart failure, established CVD, and CVD risk. The project, a public-private partnership between the regional government of Apulia, Local Sanitary Agency (ASL) of Lecce, Pfizer Italy, and Pfizer Health Solutions, United States (PHS) was implemented in the Lecce district of Apulia, Italy. The key goals of the project aimed to:

1. Demonstrate the feasibility of implementing disease and care management programs for patients with established CVD, diabetes, heart failure, or CVD risk in the Lecce ASL for possible future rollout to other areas to supplement national efforts made in this field in Italy.

2. Assess the perceptions and satisfaction of the disease management and care management services by the participating patients, doctors, and care managers.

3. Refine and customize the disease and care management intervention to fit the local environment for potential program expansion throughout the Apulia region.

The Apulia region in southern Italy was chosen as the initial implementation site for a disease and care management project for several reasons. First, with $15.2 \%$ of population over the age of $65,{ }^{3}$ Apulia had one of the highest percentages of elderly citizens in southern Italy and the prevalence of CVD and diabetes in the region were higher than in the northern regions. Additionally, patient perceptions of the quality of health care offered in the southern regions of Italy were less favorable than in other regions, with more patients electing to seek care outside of Apulia. Finally, the local region had already taken action and implemented a larger strategy aimed to improve the management of CVD-related conditions and to reduce obesity and smoking, two key CVD risk factors, among residents in Apulia.

\section{Material and methods}

Project Leonardo used a team-based approach to disease management with care managers, physicians, and specialists working together as "partners" of the patient. The project assumed the full involvement of the following professionals:

1. General practitioners (GPs) and family physicians working in a specific territory and organized in group practices.

2. Referring specialists including cardiologists and diabetologists.

3. Care managers serving as a bridge between physicians, specialists, and patients, collaborating with the patients' 
doctors while working directly with patients. All patients with established CVD, diabetes, heart failure, and CVD risk were considered for participation.

Patients participating in Project Leonardo would work with the care manager assigned to their GP or family doctor. All patients received: (a) initial and follow-up assessments conducted by the care manager in order to establish baseline measures of health measures and behaviors and provide a means for tracking patient progress during the study, (b) an individualized care plan which reflected the treatment recommendations of their doctor and specialists as well as personal health goals chosen by the patient, (c) educational materials matched to their specific conditions or risk factors, (d) assistance with service coordination including easier access to specialist care, and (e) regular, ongoing one-on-one health coaching sessions offering opportunities to address individual patient concerns and goals.

Building on the specific treatment recommendations given to patients by their doctor, meetings between the care manager and the patient could take a broad perspective and consider the medical, social, behavioral, and emotional impact that living with a chronic condition or reducing a health risk might have on the patient's quality of life. Each of the care manager's interventions were intended to increase patient empowerment, supporting patients to develop the confidence to effectively self-manage their health.

Throughout the course of treatment, the care manager would provide support to the patient in implementing actions based on the GP recommendations or in taking steps to make the lifestyle changes needed to improve health or lower health risks.

In managing patients the care managers used InformaCare ${ }^{\circledR}$ (Pfizer Health Solutions Inc, New York, NY), an evidencebased, decision support Internet tool designed to achieve better coordination of care and improved outcomes. In addition to providing an electronic patient record, InformaCare provided automatic alerts and reminders regarding the health status of individual patients, tools for monitoring behavior change, a resource library including care manager guidelines and patient educational materials, and summary reports that could facilitate specialist visits.

\section{Study design and measures}

Project Leonardo was implemented for an 18-month period. Enrollment of patients began in February 2006 and concluded in September 2007. A total of 20 GP group offices located in the Lecce district and surrounding areas, representing 83 physicians, participated in Project Leonardo, enrolling
1,160 patients. Thirty care managers were assigned to the GP groups based on the location of the office to their respective homes. In the larger GP practices, more than one care manager was assigned. Project management consisted of a program director, three program coordinators, and six nurse supervisors.

Project Leonardo was designed as an 18-month, pre-post feasibility study with data collected at baseline and at sixmonth intervals throughout the study duration (baseline, six months, 12 months, and final measures). Project Leonardo was organized to take place in the community, with services provided in the offices of general and family medicine doctors. Care managers were assigned to a GP practice and provided with an office where to meet with patients.

Care managers were provided with a home visit kit containing paper copies of the study assessments, patient education booklets and handouts, screen shots of the data fields, and feedback reports from the InformaCare software to use in the patient home.

\section{Project management team}

A project management team, consisting of a program leader, three program coordinators, and a technical resource person, was responsible for the day-to-day program operations including supervision and training of the care managers, and analysis of program operational data.

\section{Care manager supervisors}

Six head nurses chosen by the district were trained in the role of care manager supervisor. The supervisors participated in a two week "train the trainers" session which covered all of the clinical and technical aspects of the care manager curriculum including a review of the project protocol, the medical guidelines, the empowerment model, and the software system. Since the supervisors were expected to replicate the training for the first group of care managers, supervisors participated in practice teaching sessions and also reviewed specialized materials that would support their role in providing both clinical and administrative supervision.

\section{Care manager hiring and training}

Based on the project enrollment targets, projected caseloads, and available resources, thirty full time nurse care manager positions were created by the local health authority (the ASL) and assigned to work in group physician practices. Care management positions were open to trained nurses who had been working as hospital or home care nurses in the district in various capacities. A job profile describing the requirements for the care manager position, along with 
an interview guide for the project coordinators were developed to assist with hiring decisions. Once hired, the care managers participated in a multi-faceted, two week training to prepare them for their role as care manager. A "train the trainer" approach was utilized, with the project leader and coordinators, PHS, and Pfizer Italia delivering the training to designated local trainers.

The initial training was followed by focused training meetings every two weeks for three months coordinated by the project coordinators, and an additional week of retraining on areas of need identified by project leaders and the care managers. In addition, administrative and clinical issues were addressed in weekly group meetings attended by the care managers, coordinators, and project leader.

Further, individual supervision sessions with the project leader, coordinators and Supervisors were available on an as needed basis for the care managers to assist them in working with patients. PHS also provided additional training support, focusing primarily on care manager coaching skills on an as-needed basis throughout the project.

\section{Patients inclusion/exclusion criteria}

Project Leonardo targeted patients with the following four health conditions or risks:

1. Established CVD

2. CVD risk

3. Diabetes

4. Heart failure

Patients were excluded from participation in Project Leonardo if they:

- Refused or did not sign the consent form or revoked consent.

- Were unable to communicate in Italian.

- Were unable to communicate over the telephone.

- Had one or more of the following complex medical conditions: End-stage renal disease, HIV/AIDS, sickle cell anemia, transplant recipient, active psychoses, hemophilia, advanced cirrhosis, spinal cord injury, continuous drug dependence, congenital heart disease, current pregnancy, congenital adult cardiopathy, terminal cancer, moderate to severe dementia, life expectancy less than one year.

\section{Enrollment process}

As eligible patients were identified, they were opportunistically approached by their GP as they came into the GP office for a routine appointment. GPs provided a brochure describing Project Leonardo and invited patients to participate. For patients who accepted the invitation, the GP would obtain written consent. Once the patient consented, the GP introduced the patient to the care manager who began the enrollment process. Care managers obtained demographic and contact information from patients and enrolled them into the program via the InformaCare automated software application. Care managers would also explain details of the project, answer any patient questions, and set up a follow-up appointment to complete the initial assessment.

\section{Description of the sample}

The sample consisted of 1,160 patients, 553 females and 607 males, ranging in age from 19 to 96 years old. The average age of patients was 64 years (standard deviation of 11.12 years), with $83 \%$ of women and $82 \%$ of men between 50 and 79 years old. All patients $(1,160)$ were enrolled in the CVD module, with 912 patients (78.6\%) diagnosed with established CVD and 236 (20.3\%) classified as CVD risk. Of the 533 patients enrolled in diabetes, there were near equal numbers of women $(269 \%$ or $50.4 \%)$ and men $(264 \%$ or $49.5 \%)$. For the 192 patients with heart failure, $39.5 \%$ or 76 were women and $60.4 \%$ or 116 were men. Over half of the patients in the study $(649 \%$ or $56 \%)$ were diagnosed with more than one of the primary study conditions (CVD, diabetes, heart failure). Of those with more than one chronic condition, 573 patients had two conditions and 76 patients were diagnosed with all three: CVD, diabetes, and heart failure. With regard to lifestyle habits and risk factors, at baseline $158 \%$ or $13.6 \%$ of the patients reported that they were current smokers, 664 were not ready to take action to increase their physical activity level, 622 patients were not ready to make any changes to improve their diet and nutritional habits, and $85.3 \%$ of the sample had a body mass index (BMI) in the overweight or obese range. Of the 1,160 patients who participated, 485 patients $(41.8 \%)$ received between 9 and 12 months of the intervention, 426 (36.7\%) between 13 and 18 months, and 249 (21.4\%) between 19 and 21 months.

\section{Initial assessment}

After enrollment, an initial assessment was conducted by the care manager to gather data and information from the patient. This served as both the baseline data to track patient progress against, as well as information from which the care manager would collaborate with both the patient and the GP to develop an individualized care plan. The following information was collected: medical history, diagnoses, medical procedures, illness complications, utilization (hospital and 
emergency visits), allergies, medications, physical measures, labs, immunizations, screenings, and health behaviors (including smoking, diet, weight, physical activity, and selfmanagement skills).

As part of the study design, care managers also repeated the initial assessments with all enrolled patients at six-months and one-year follow-up.

\section{Level of care}

Once the patient information from the initial assessment was recorded in InformaCare, the system would generate a suggested level of care (LOC), specific to the patient. LOC for Project Leonardo was developed to assist the care manager in stratifying patients. Participants were stratified into one of three or four LOC based on current and prior condition severity, lifestyle behaviors, and co-morbidities. The GP, in cooperation with the care manager, would review the patient's system-generated LOC and validate it by applying their clinical judgment and knowledge of the patient, modifying it when necessary so that the LOC was clinically appropriate.

\section{Frequency of contact between care managers and patients}

Based on the patient LOC, care managers were instructed to use the recommended frequencies as a minimal guideline for the amount of contact to have with patients. It was expected that the care manager would consider the patient's preferences and requests, and if possible accommodate the patient needs.

\section{Intervention description}

All interventions with patients in Project Leonardo were based on a patient empowerment approach designed to support the promotion of self-management. The empowerment model required that patients be viewed as experts on their own lives and responsible for their own health. ${ }^{15}$ In order to support patient self-management, patients' perspectives on their conditions, their goals, expectations, and needs were considered as the primary focus of the treatment goals and management activities. The model of care stressed the importance of the partnerships between members of the patient's health team (the patient's doctor, the specialists, the nurse care manager, and the patient). The care plan was individualized and the central focus of the health team's work where patients were considered the most important member, the one who would carry out the necessary actions.

\section{Care coordination}

Throughout the project care managers helped to coordinate patient care by assisting the patient in arranging for visits to specialists and supporting the patient in the use of other community resources. The care manager also helped to manage the patient follow-up appointments with the GP based on the LOC and to schedule case conferences based on the individual patient's needs. A special report was created by the local project team to provide the specialist with an update on the patient status and progress as well as the current care plan focus. ${ }^{7}$

\section{Assessment of "Leonardo" feasibility and effectiveness}

Leonardo's project tried to underline the importance of new professional care figures able to fill the great deficiency in the health care system concerning the lack of a link between patients and their own illnesses. In order to assess the feasibility and effectiveness of Project Leonardo, we used the SF-12 questionnaire, ${ }^{16}$ to assess the quality of life in patients suffering from, in this case, CVD and diabetes. The SF-12 health survey is comprised of 12 questions from the SF-36 health survey. These include two questions concerning physical functioning, two questions on role limitations because of physical health problems, one question on bodily pain, one question on general health perceptions, one question on vitality (energy/fatigue), one question on social functioning, two questions on role limitations because of emotional problems, and two questions on general mental health (psychological distress and psychological well-being). Comparative criteria by Ware et $\mathrm{al}^{17}$ pointed out the normal value of the latter score system in Italy. This made us realize the kind of impact our study could have on health care improvement from the standard value of the Italian population.

In order to evaluate the strict adherence to therapy schemes, we adopted Morisky compliance scale, ${ }^{18}$ a commonly used adherence screening tool composed of four yes/no questions about past medication use patterns which is quick and simple to use during drug history interviews. We gave this test at the beginning of our study and during the development of the study, during care manager activities, to try underline the differences between the initial scores and the scores as the study progressed. No criteria were pointed out before starting the exam because we wanted to illustrate the importance of the care manager in improving the beginning level of adherence to the therapy of a patient.

The blood pressure, cholesterol, and glycosylated hemoglobin blood level of each patient was taken in order to reach 
a complete panel of the health improvement of the individuals studied. In particular, we wanted to analyze the number of patients able to reach normal or even optimal standard level of these parameters:

- Routine assessment and monitoring of blood pressure at home between visits to the doctor may be an important component of CVD management. Many patients with CVD will be completely asymptomatic. Blood pressure measurement in the home setting may be beneficial in providing information on response to blood pressure medication, improving patient adherence, and in evaluating "white-coat" hypertension, a condition noted in patients whose blood pressure is consistently elevated in the doctor's office or clinic, but normal at other times.

- Self-monitoring of blood glucose (SMBG) provides a mechanism for patients to closely monitor glucose levels on a frequent basis. Self-monitoring alerts the patient to impending episodes of hypoglycemia or ketoacidosis, and the impact diet and physical activity has on glucose control. SMBG also provides guidance for medication adjustment.

- Worsening heart failure may be triggered by fluid overload and may manifest as trouble breathing, ankle swelling, and/or weight gain. Early detection and management of symptoms of worsening heart failure can help avoid a hospitalization for a patient.

\section{Statistical analysis}

The data are given as mean values or percentages. Withingroup comparisons were made using the Student's $t$-test for dependent variables. Frequencies were compared using McNemar's test (paired proportions).

\section{Results}

Disenrollment and drop-out of patients was low throughout the study. Of the 1,160 patients enrolled in Project Leonardo, only 54 patients were disenrolled, so the drop-out rate did not affect results and scope of the project.

Project Leonardo was able to successfully implement a consistent and standardized set of guidelines ensuring that the necessary tests and services recommended for patients with CVD, diabetes, and heart failure were provided on schedule. The project exceeded the goal that $70 \%$ of patients would receive recommended tests and services as appropriate. Testing provided opportunities to keep the patient's medical conditions and risk factors "in check" and to have current clinical information giving an indication of the patient status and allowing doctors to provide interventions in a timely and effective manner. Taking this proactive approach offered an opportunity to lessen the need for more costly treatments and reduce the negative impact of the condition on the patient. Information on recommended tests and services was shared by the all members of the health team and included education of patients about the rationale and timing for tests and the impact of test results on their health management. In fact, care managers were commended by the some of the doctors as the "guardians" of the testing schedule since it was common for the care manager to provide this type of monitoring, and this helped keep patients informed as well served to remind doctors when the patient needed additional tests. Lessening the gap between the recommendations of clinical guidelines and the care patients actually received throughout the Project can be regarded as one of the major achievements of Leonardo.

Patients showed significant improvements in many of the psychosocial and behavioral variables. Patients increased in their self efficacy, coping, and ability to access social support. Patient self-monitoring behavior increased dramatically during the study period with an additional $20 \%-27 \%$ of patients in each condition taking a more active role in the management of their condition. In addition, there were substantial numbers of patients who shifted from only doing the activity to becoming independent showing an increase in their self-management skills.

With regard to lifestyle behaviors, there is evidence that patients were taking more action to adopt healthier eating habits, to increase their amount of physical activity, and higher numbers of patients who were not just thinking about quitting smoking, but were actually in the process of doing it. In fact, we pointed out that a healthy diet became the new nutrition trend in $41 \%$ of new patients: during the follow-up period, we saw an increase from 413 (39.4\%) to 847 persons $(80.7 \%)$ of those who had adopted a good quality diet. Moreover, we all know the importance of being physically active, especially for individuals suffering CVD and diabetes. The care manager helped in improving intensity of physical training: 112 patients improved their baseline physical state, being able to shift from a starting "low intensity" level of exercises to a "medium" level; seven had been able to pass from a "medium" level to a "high" level, and five patients managed to shift from a "low intensity" level to the "high" level. There was also a statistically significant increase in the number of days per week employed for physical training, from 2.53 to 4.18 days $(P<0.0001)$. There was also an increase in time spent doing physical activity, from 19.87 to 32.90 minutes $(P<0.0001)$ per session. 
Several key clinical goals with the potential to lower the risks associated with CVD or diabetes showed significant improvements during the study. The results show that there was statistically significant change in BMI, low-density lipoprotein (LDL), systolic blood pressure, and total cholesterol which had important clinical significance in that the improvements resulted in an increase of the percentage of patients who met the recommended target goals for those values. At the initial assessment, the population studied showed that: four patients were "underweight" (BMI < 18.5); 158 were "healthy" (BMI < 25); 420 were "overweight" $(\mathrm{BMI}<30)$ and 522 were "obese" (BMI > 30). At the final assessment, 35 patients had shifted from "obese" to "overweight", and 40 individuals had shifted from "overweight" to "healthy." According to cholesterol blood level, our aim was to reduce such a blood parameter to a level considered normal for a patient at risk of a major cardiovascular event:

1. Patients with CVD or diabetes: $\mathrm{LDL}<100 \mathrm{mg} / \mathrm{dL}$ and total cholesterol $<175 \mathrm{mg} / \mathrm{dL}$

2. Patient at risk for CVD, but not diagnosed: $\mathrm{LDL}<115 \mathrm{mg} / \mathrm{dL}$ and total cholesterol $<190 \mathrm{mg} / \mathrm{dL}$.

All patients should reach an high-density lipoprotein (HDL) level $<40 \mathrm{mg} / \mathrm{dL}$ in males and $<50 \mathrm{mg} / \mathrm{dL}$ in females, and triglycerides $<150 \mathrm{mg} / \mathrm{dL}$. At the end of the study, all parameters were reduced by at least $10 \%-20 \%$ and HDL level did not significantly increase.

Of key importance are the changes observed in blood pressure. Our goal was to reach a normal value, in particular: $<140 \mathrm{mmHg}$ systolic and $<90 \mathrm{mmHg}$ diastolic for most patients, $<130 \mathrm{mmHg}$ systolic and $<80 \mathrm{mmHg}$ diastolic for diabetes, and $\leq 125 \mathrm{mmHg}$ systolic and $<75 \mathrm{mmHg}$ diastolic for patients with chronic kidney disease. There was a significant decrease in both diastolic and systolic blood pressure values $(P<0.0001)$ from initial to final measurements. The decrease in systolic values has important clinical significance and when combined with the additional changes in lipid profile and weight, the impact for individual patients who experienced changes in multiple areas may be even more important to their health than changes in any one variable. Furthermore, because there was little change in the type or amount of medication used to treat blood pressure or cholesterol during the study, it is likely that the changes in the clinical indicators reflect the efforts and changes in behavior that were accomplished by the patients eating healthier diets, increasing physical activity, or quitting smoking..$^{19,20}$

Moreover, SF-12 measures of the physical and mental health status showed that despite the impact of multiple serious physical illnesses, patients were able to maintain a positive mental health status and even increase their sense of vitality over the course of the study. In fact, at the follow-up the average score obtained 7.99 points above the national normal value (47.6) and 5.28 points above the starting score of the population studied. This increase in SF-12 score is important because it shows a real improvement of physical and mental state of the patients followed by a care manager.

This means that efforts to increase patient empowerment and participation should be continued for all patients, regardless of the seriousness of their condition, because even small changes may be meaningful to the patient's overall sense of well-being.

Finally, high satisfaction ratings based on survey results from physicians, care managers, and patients showed unanimous agreement regarding the project's positive impact on patient health and self-management and unanimously attributed the positive outcomes to the strong "partnership" between the care manager and the patient, and the effective collaboration between the physician and the care manager. The care manager is the key to realizing the goal of Project Leonardo. A Care Manager must understand diagnoses and the therapeutic goals that physicians manage to reach with drug or more invasive techniques, and be able to explain them to the individuals in lay terms in order to help patients understand their illness, symptoms, and signs. The care manager keeps the patients informed about medical appointments, informs them of rehabilitation schemes, and shows patients how to implement behavior changes. A care manager must have skills in understanding people and be sympathetic to their patients' problems so that patients can complete the care program with optimal results.

\section{Discussion}

The Project Leonardo intervention was expected to impact patient health from several interrelated perspectives. First, the project scientific committee and doctors participating in the project worked together and agreed to implement one set of guidelines and recommendations for each condition in a consistent way throughout the study period, ensuring that all aspects of the patient health would be monitored and evaluated. Second, as the empowerment model was introduced and implemented, patients would be activated to participate more fully in their health care. This shift would allow patients to use the "partnership" with their doctor and care manager as a way to gradually build the self-confidence and the motivation to make behavior changes that could 
impact their health. As patients made changes in their health behaviors, improving nutrition, increasing physical activity, increasing self-monitoring behaviours, and becoming more adherent to testing and treatment recommendations, their clinical indicators (such as LDL levels, BMI rating, blood pressure values) also change in a positive way. Overall, these changes have the potential of reducing hospitalizations and emergency care costs as well as leading to greater satisfaction among patients, doctors, and care managers.

Since most GP group practices did not have nurses as part of their staff, prior to Project Leonardo, and it was common for GPs and family doctors to work alone, each practice was expected to make the necessary arrangements to accommodate the care manager. In this way, Project Leonardo offered an opportunity for doctors and nurses to examine the possibilities of direct collaboration about patient care. While it was expected that most meetings between the care manager and the patient would occur at the GP practice, care managers were also encouraged to provide home visits for patients who could not come to the office due to illness or had other physical or situational barriers that made it difficult for them to attend meetings in the GP office.

\section{Conclusion}

In conclusion, Project Leonardo demonstrated the feasibility of incorporating care managers (specially trained nurses) into the health care system to support GPs and specialists in the management of patients with CVD, diabetes, heart failure, or CVD risk. Care managers worked directly with individual patients, helping them to make lifestyle changes, monitoring their conditions, and providing the necessary information and advice to promote patient empowerment, enhance self-management skills, and achieve better compliance with care recommendations. This model resulted in a tangible improvement in the clinical parameters of the patients enrolled who thus achieved better control of their disease. In such a setting, the combined efforts and networking of all the persons involved created a strong collaborative "health team" approach spanning 20 group practice offices and involving 83 GPs, 30 care managers, 10 specialists, and 1,160 patients.

\section{Acknowledgments}

The authors wish to thank JA DeBonis, Andrea Musilli, Marina Panfilo, (PHS), ${ }^{22}$ and Piero Guida for their contributions to this paper.

\section{Disclosures}

The authors report no conflicts of interest in this work.

\section{References}

1. Murray CH, Lopez AD. The Global Burden of Disease: A Comprehensive Assessment of Mortality and Disability from Disease, Injuries, and Risk Factors in 1990 Projected to 2020. Cambridge, MA: Harvard School of Public Health; 1996.

2. Davies E, Higgins I, editors. Better Palliative Care for Older People. Geneva, Switzerland: World Health Organization; 2004.

3. Bonow RO. Prevention of cardiovascular disease: challenges and opportunities. Ital Heart J. 2004;5(Suppl 6):6S-8S.

4. Bonow RO, Smaha LA, Smith SC, et al. World Heart Day 2002. The international burden of cardiovascular disease; responding to the emerging global epidemic. Circulation. 2002;106:1602-1605.

5. Leal J, Luengo-Fernandez R, Gray A, et al. Economic burden of cardiovascular disease in the enlarged European Union. Euro Heart $J$. 2005;27(13):1610-1619.

6. Mola E. Dalla compliance all'empowerment: due approcci alla malattia. In: Dipartimento di Filosofia e Scienze Sociali, Lecce. Quaderno di Comunicazione: Fiducia Sicurezza. Roma, Italia: Meltemi Editore; 2006: 99-107.

7. Anderson RM, Funnell MM. Patient empowerment: myths and misconceptions. Patient Edu Couns. In press 2009. doi:10,1016/ j.pec.2009.07.025.

8. Bodenheimer T, Lorig K, Hlasted H, Grumback K. Patient selfmanagement of chronic disease in primary care. JAMA. 2002;288(19): 2469-2475.

9. Azarmina P, Prestwich G, Rosenquist J, Singh D. Transferring disease management and health promotion programs to other countries: critical success factors. Health Promot Int. 2008;23(4):372-379.

10. Lorig K. Self-management education: more than a nice extra. Med Care. 2003;(6):699-701.

11. Wagner EH. Chronic disease management: what will it take to improve care for chronic illness? 1998. Available from: http://www.acponline. org/clinical_information/journals_publications/ecp/augsep98/cdm.htm. Accessed 2010 March 29.

12. World Health Organization. Innovative Care for Chronic Conditions: Building Blocks for Action. Geneva, Switzerland: World Health Organization, 2002.

13. Lorig K, Holman H, Sobel D, Laurent D, Gonzalez V, Minor M. Living a Healthy Life with Chronic Conditions. Self-Management of Heart Disease, Arthritis, Diabetes, Asthma, Bronchitis, Emphysema, and Others. Boulder, CO: Bull Publishing Company; 2000.

14. Fischer D, Stewart AL, Anita L, et al. Capturing the patient's view of change as a clinical outcome measure. JAMA. 1999;282(12):11571162.

15. Mola E, DeBonis J, Giancane R. Integrating patient empowerment as an essential characteristic of the discipline of general practice/family medicine. Eur J Gen Pract. 2008;26:1-6.

16. Hopman WM, Harrison MB, Coo H, Friedberg E, Buchanan M, VanDenKerkhof EG. Associations between chronic disease, age, and physical and mental health status. Chronic Dis Can. 2009;29(3): 108-116.

17. Ware J, Gandek B, Kosinski M, et al. The equivalence of SF-36 summary health scores estimated using standard and country-specific algorithms in 10 countries: results from the IQOLA project. $J$ Clin Epidemiol. 1998;51(11):1167-1170.

18. Shalansky SJ, Levy AR, Ignaszewski AP. Cardiology: self-reported Morisky score for identifying nonadherence with cardiovascular medications. Ann Pharmacother. 2004;38(9):1363-1368.

19. Berg IK, Reuss NH. Solutions Step by Step: A Substance Abuse Manual. New York, NY: W.W. Norton \& Co.; 2000. 
20. Britt E, Hudson SM, Blampied NM. Motivational interviewing in health settings: a review. Patient Edu Couns. 2004;53(2):147-155.

21. Hunt SA, Baker DW, Chin MH, et al. ACC/AHA guidelines for the evaluation and management of chronic heart failure in the adult: a report of the American College of Cardiology/American Heart Association Task Force on practice guidelines. Circulation. 2001;104:2996-3007.
22. Azarmina P, Prestwich G, Rosenquist J, Singh D. Transferring disease management and health promotion programs to other countries: critical success factors. Health Promot Int. 2008;23(4):372-379.

Vascular Health and Risk Management

\section{Publish your work in this journal}

Vascular Health and Risk Management is an international, peerreviewed journal of therapeutics and risk management, focusing on concise rapid reporting of clinical studies on the processes involved in the maintenance of vascular health; the monitoring, prevention and treatment of vascular disease and its sequelae; and the involvement of metabolic disorders, particularly diabetes. This journal is indexed on PubMed Central and MedLine. The manuscript management system is completely online and includes a very quick and fair peer-review system, which is all easy to use. Visit http://www.dovepress.com/ testimonials.php to read real quotes from published authors.

Submit your manuscript here: http://www.dovepress.com/vascular-health-and-risk-management-journal 